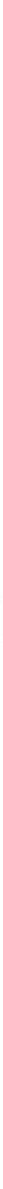

Drug Regulatory Affairs

Formulation Including

Pharmacokinetic Aspects

International Pharmaceutical Issues

Good Manufacturing Practice

Quality Control 



\title{
A NOTE ON THE TRANSDERMAL DELIVERY OF CLENBUTEROL
}

\section{Achim Göpferich and Geoffrey Lee $^{1}$}

Institute for Pharmaceutical Technology and Biopharmaceutics, Heidelberg University, Germany.

\begin{abstract}
A modified diffusion/compartmental model has been used to simulate the transdermal uptake of clenbuterol from a matrix-type delivery device. The application of a fresh device every 7 days was found to produce a pseudo-steady state drug plasma profile after approx. three changes of device. Of the matrix properties, only drug loading had substantial effect on the drug plasma profile.
\end{abstract}

\section{$\underline{\text { INTRODUCTION }}$}

Drugs having long pharmacokinetic half-lives are considered to be unsuitable for transdermal application, owing to the prolonged times $\left(\mathrm{t}_{\max }\right)$ necessary before drug plasma levels achieve a pseudo-steady state (1). An example is the $\beta-2$ adrenergic agonist, clenbuterol, which has a half life of

1Correspondence: Prof. Geoffrey Lee, Institut für Pharmazeutische Technologie und Biopharmazie, Im Neuenheimer Feld 366, 69(0) Heidelberg, Germany. 
20-35 $\mathrm{h}$ after i.v. injection (2). The topical application of this drug from a matrix-type transdermal device has been previously analyzed using a combined diffusion/compartmental model for transdermal drug uptake. Application of the model to some in vivo data obtained for a single device worn for 7 days showed that a $t_{\max }$ of some 21 days could be expected with this drug (3). Simulations showed that this extended $t_{\max }$ could not be reduced substantially by altering either the properties of the matrix device or the permeability of the stratum corneum with a permeation enhancer. Further simulations with a model for transdermal drug uptake from a membrane-controlled-type device (4) illustrated the possibility of reducing $\mathrm{t}_{\max }$ by incorporating a 'loading dose' of drug into the rate-controlling membrane.

Matrix-type devices are, however, advantageous by virtue of their simple construction. For this reason, our attention turned to an examination of the repeated application of such a device over a prolonged period. We wished to determine if an acceptable drug plasma profile could be obtained in this way by varying the properties of the device or those of the stratum corneum. The results are presented in this paper.

\section{THEORY}

For this study we took a combined, non-steady state diffusion/compartmental model for transdermal drug delivery from a matrixtype device (3). To allow for the effects of application of a fresh device every 7 days, it was necesssary to introduce a parallel pathway into the model (Fig. 1). Thus, pathway I represents uptake of drug from the current matrix, whereas pathway II allows for the drug residue still existing in the 


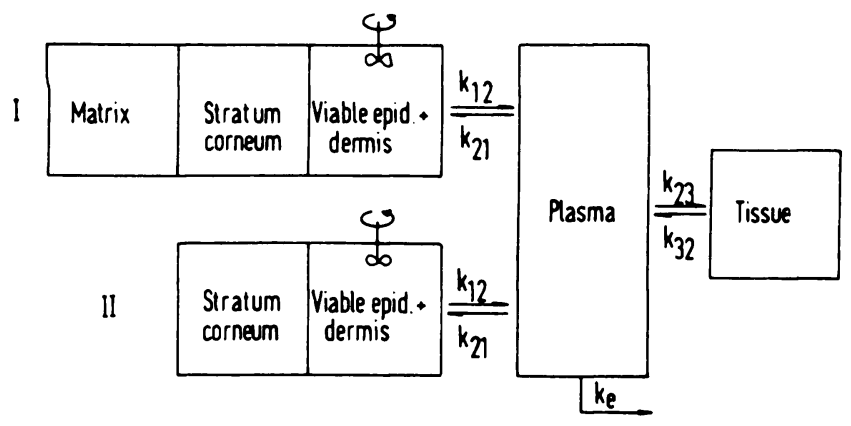

FIGURE 1

Modified diffusion/compartmental model for repeated application of a matrix-type device.

system from the previous matrix. With the help of this model it was possible to produce simulated plasma profiles for clenbuterol in a matrix prepared from Eudragit NE30D. Values for the various system constants were taken from the literature $(3,5)$. The effects on the plasma profiles of changing drug loading of the matrix, matrix thickness, drug diffusivity in the matrix, and drug diffusivity in the stratum corneum were simulated. In this way, we hoped to determine if the transdermal application of clenbuterol from such a device was at all feasible.

\section{$\underline{\text { RESULTS AND DISCUSSION }}$}

The influence of drug loading of the matrix on the plasma profile is simulated in Fig. 2. As a result of the non-sink, non-steady state nature of the model, the plasma profile for each application of a matrix shows a lag phase, after which the drug concentration climbs to a maximum, and then declines. A 'pseudo-steady state' plateau is achieved during application of 


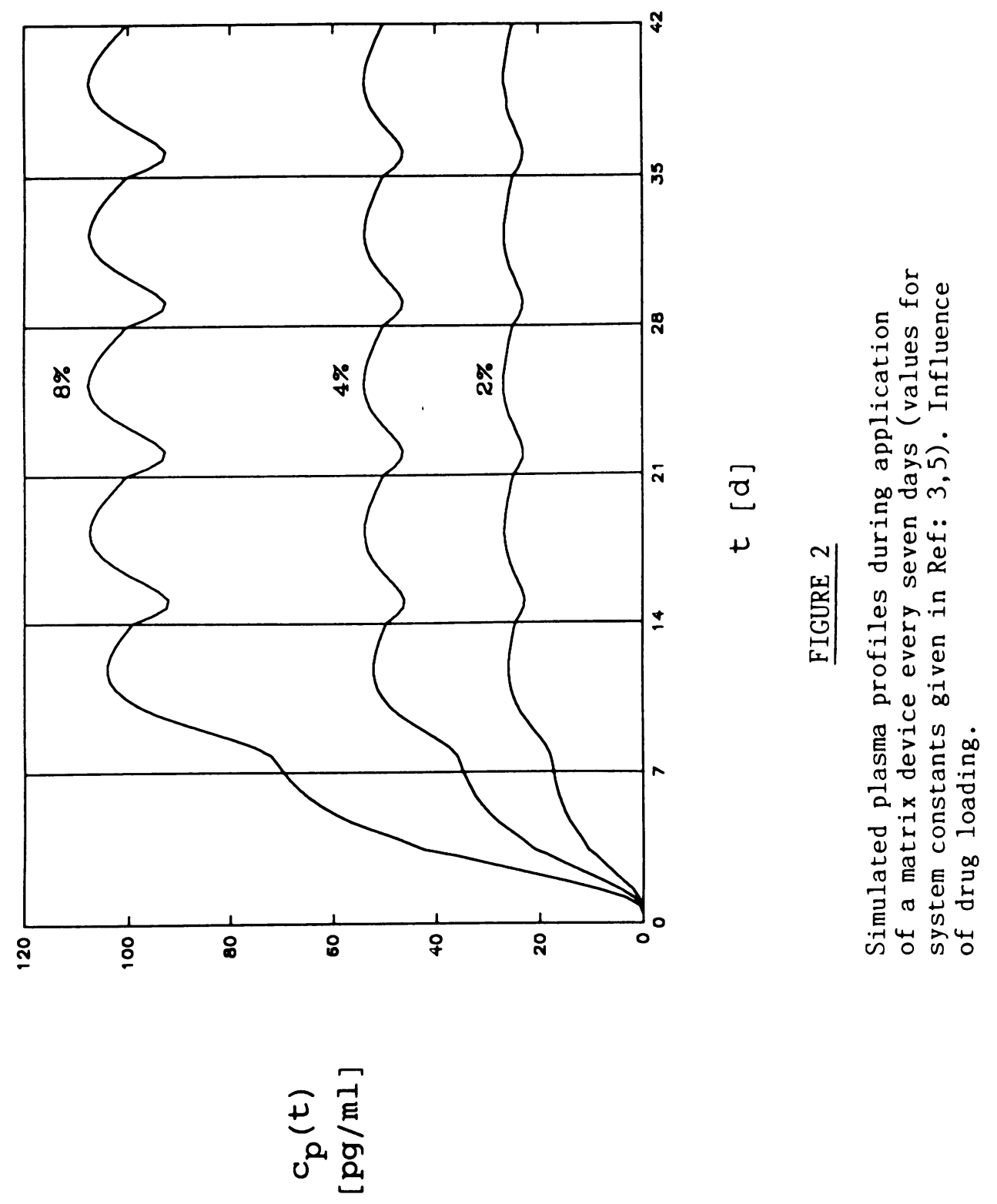



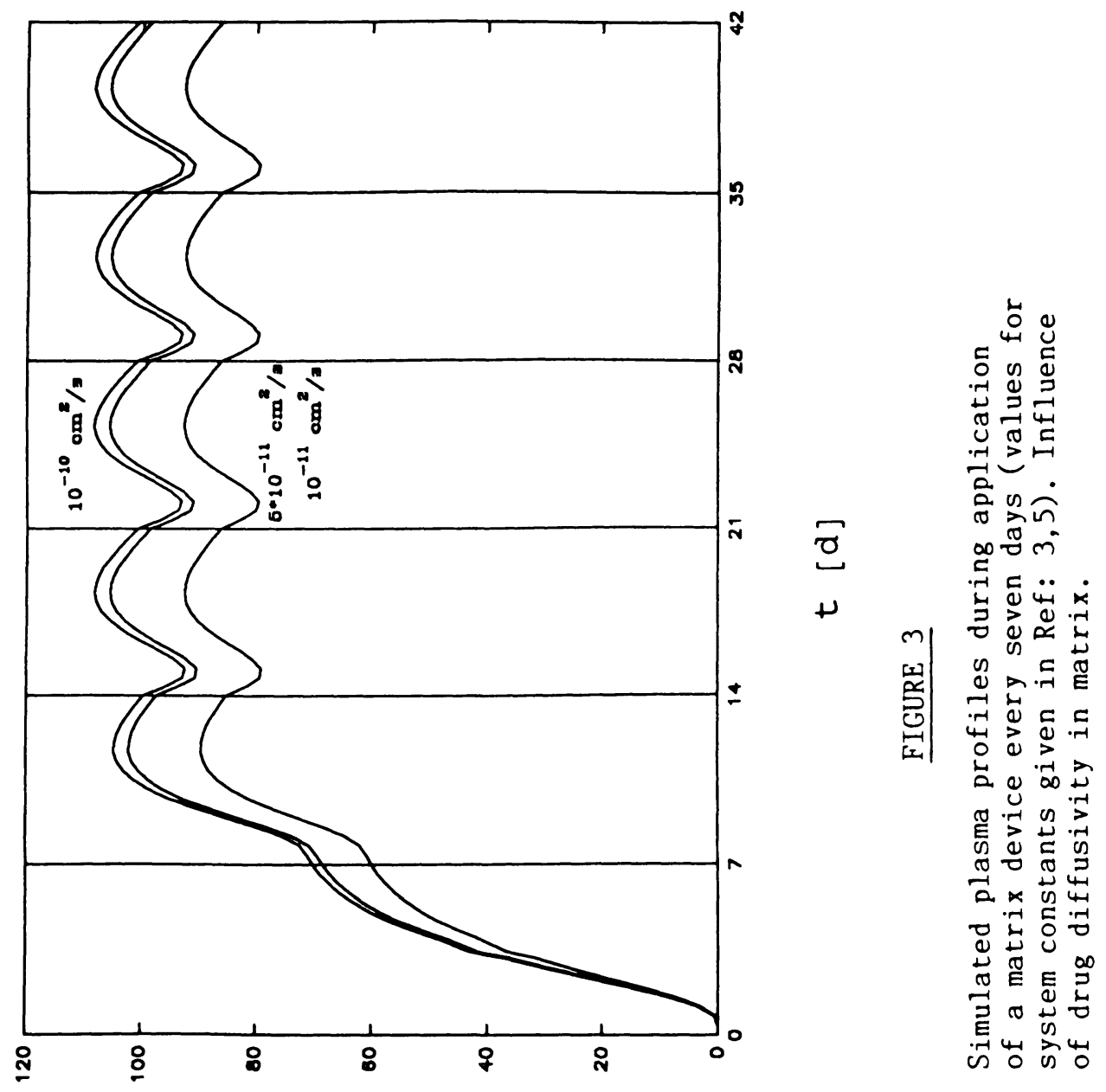

$$
\begin{aligned}
& \text { 퐅 } \\
& \text { ن ह }
\end{aligned}
$$



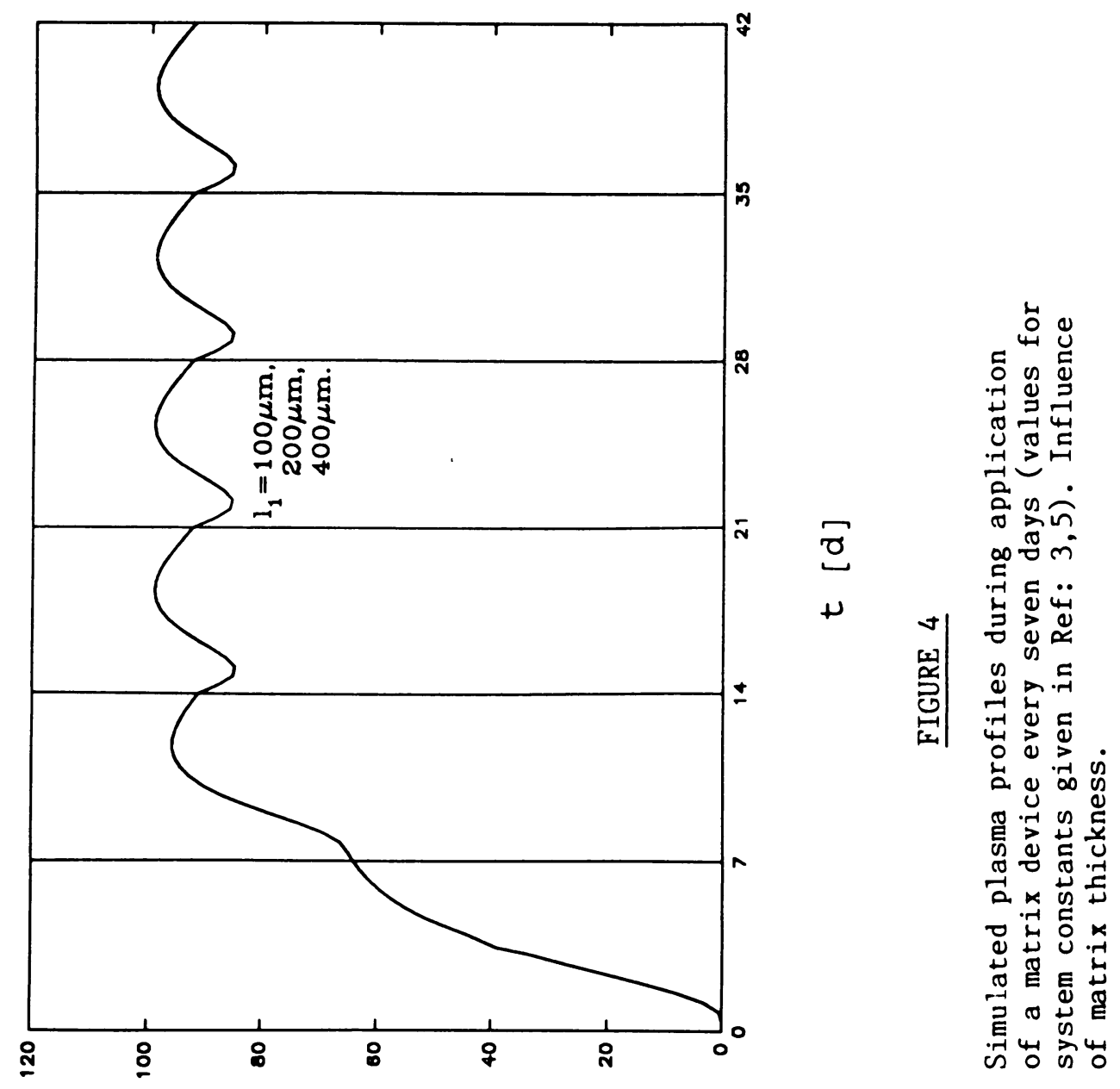

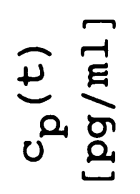




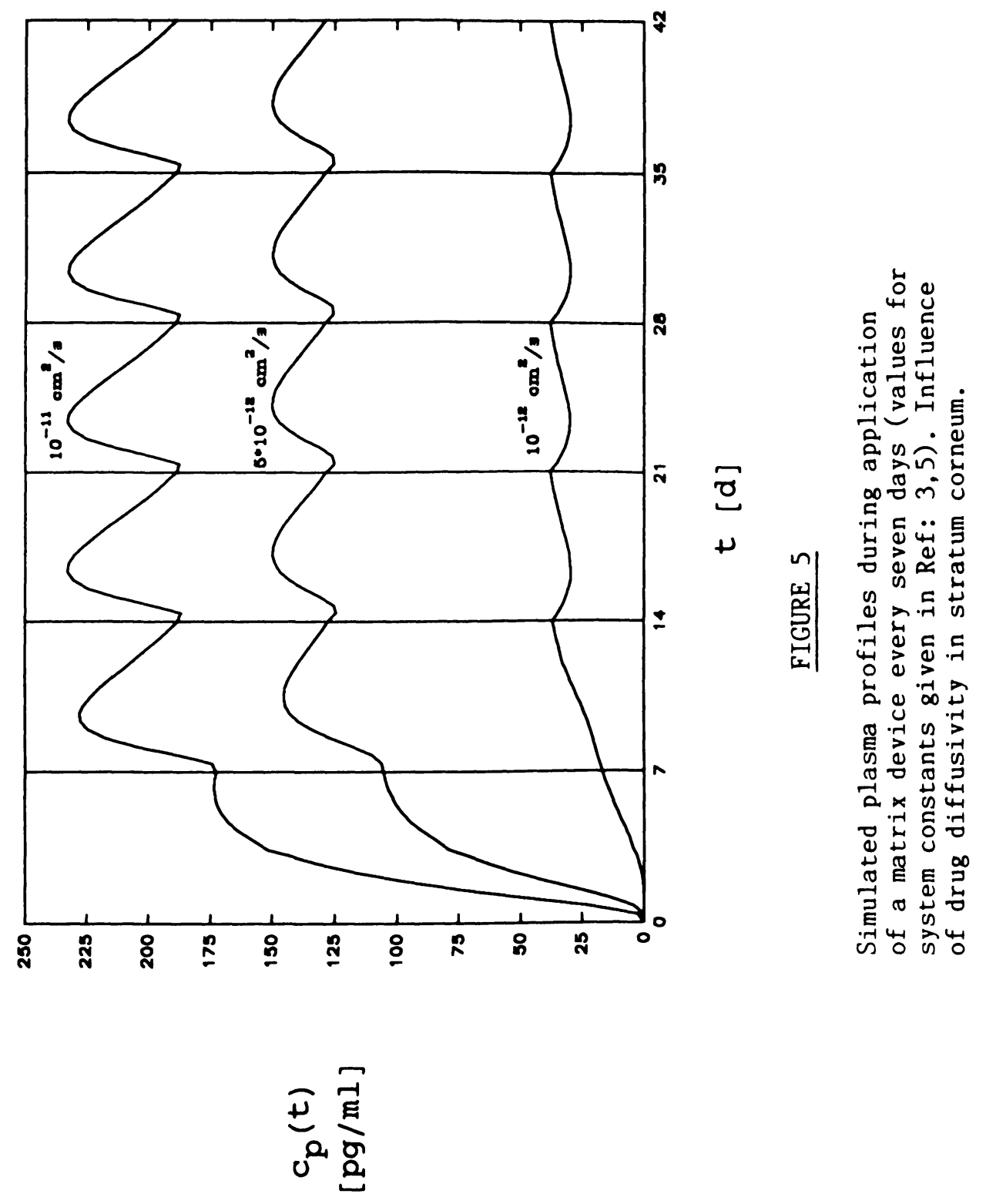


the third device. The oscillation of the plasma profile is damped in this phase, owing to the existence of a drug 'depot' within the stratum corneum. This reduces the influence of the lag time for drug passage through the stratum corneum. The drug loading clearly has a large influence on the plasma profile. With $8 \%$ loading, the plasma profile lies well within the required therapeutic concentration for clenbuterol of approx. $100 \mathrm{pg} / \mathrm{ml}(5)$.

The diffusivity of the drug within the device has much less influence on the plasma profile (Fig. 3). Indeed, an increase of an order of magnitude hardly changes the profile. The thickness of the device has no discernable influence on the profile (Fig. 4), as known from previous work $(3,6)$. The only device property which can effectively be used to adjust the plasma profile is, therefore, drug loading. This will, however, be limited by the solubility of the drug in the matrix.

The influence of diffusivity within the stratum corneum on the plasma profile (Fig. 5) shows clearly that the complete process is strongly dependent on the properties of this membrane. A change of an order of magnitude has great influence on the plasma profile. This could clearly be a source of considerable variation in plasma profiles between patients or between different body sites.

\section{ACKNOWLEDGEMENT}

Thanks to Boehringer Ingelheim KG for its financial support of this project.

\section{REFERENCES}

(1) R. Guy and J. Hadgraft, Transdermal Drug Delivery, Marcel Dekker Inc., p. 61 (1989). 
(2) I. Yamamoto and K. Iwata, J. Pharmacobiodyn., 8, 385 (1985).

(3) A. Göpferich and G. Lee, Int. J. Pharm., 71, 237 (1991).

(4) A. Göpferich and G. Lee, Prediction of Percutaneous Penetration, II, in press (1991).

(5) A. Zimmer and A. Bücheler, Drug Res., 26, 1447 (1976).

(6) R. Guy and J. Hadgraft, Int. J. Pharm., 6, 321 (1980). 